\title{
Near Field Corrections for Coded Aperture Imaging in Scintimammography
}

\author{
M. A. Alnafea, K. Wells, M. Guy and N. M. Spyrou
}

\begin{abstract}
In this work we study the form of artifacts arising with near-field imaging geometries associated with using coded apertures for Scintimammography (SM) using a combination of Monte Carlo Simulation (MCS), Pseudo-Ray Tracing (PRT) and a new, but simple approach called binary mask shift. The latter method predicted the shape artifacts that are due to off-axis sources and finite size of the object but ignores the effect of varying the angle of incidence of the gamma-rays. The background artifact pattern produced by uniform 2D and 3D source objects of different sizes using a PRT method compared with the corresponding data obtained with MCS suggest that both methods produce striking similarities. From these results we are encouraged to believe that the so-called near field distortion observed with distributed planar and 3D sources, as might be found in SM using coded apertures, can be easily predicted and corrected.
\end{abstract}

\section{INTRODUCTION}

$P$ LANAR scintimammography (SM) is usually performed with a collimator-based gamma camera employing ${ }^{99 \mathrm{~m}} \mathrm{Tc}$ labeled Sestamibi. As an alternative approach to dedicated instrumentation development [1-3], we have been investigating $[4,5]$ the application of a Modified Uniformly Redundant Array (MURA) planar Coded Aperture (CA), instead of using a collimator, coupled to standard clinical gamma camera for breast tumour imaging. This is particularly attractive at General/County Hospital level, where the cost of running an additional dedicated imaging system may be prohibitive. Moreover, CA imaging is well suited for detecting faint point like objects $[6,7]$; thus $\mathrm{CA}$ imaging appears well matched to the imaging objectives in SM. Our recent work [5] suggests a more promising performance for the CA system is obtained with proper shielding from other non-specific background tracer uptake to which CA systems are susceptible due to the open field geometry. However, the locally generated artifacts arising from the imaging geometry of a hot source in a warm background, offset and degrade the decoded image and to our knowledge, there has not yet been a systematic study and subsequent analysis of such imaging artifacts in this domain.

Previous studies $[8,9]$ in this area have made significant strides in addressing the artifacts induced by the inherent near-

Manuscript received November 17, 2006.

M.A. Alnafea, K. Wells, and N.M. Spyrou are with School of Electronics \& Physical Sciences, University of Surrey, Guildford, Surrey, GU2 7XH, UK (phone: 441-483-686036; fax: 441-483 686031; e-mail: m.alnafea@ surrey.ac.uk).

M. Guy is with Department of Nuclear Medicine, Royal Surrey County Hospital, Guildford, Surrey, UK, GU2 7XH (phone: 441-483-406709; fax: 441-483 406702; e-mail: matthew.guy@nhs.net). field geometry. They have suggested [8] that factors such as varying the incident angle of gamma-rays, which adds an intensity modulation to the projection of the mask pattern, finite thickness of both the object and the mask are the most serious causes of such artifacts.

In this work we complement previous work by investigating in a more intuitive way some of the problems associated with the use of MURAs-CA (or patterns based on MURAs such as No-Two-Holes-Touching, NTHT,) in the near-field imaging geometries when imaging 2D and 3D objects. We focus on understanding and predicting the form and the components of the artifacts arising when using CA for SM imaging. We then implement correction methods in order to alleviate these imaging artifacts and consider the improvement in ContrastNoise-Ratio with and without the proposed corrections.

The remainder of this paper provides a description of the three different methods undertaken to investigate this proposition. Starting with the basic theoretical principle of CA imaging, we then provide a description to the different geometries used. A set of near field artifact corrections are presented and then applied to the 3D data of a hot lesion in warm background.

\section{USE OF CA IMAGING IN SCINTIMAMMOGRAPHY}

Conventional gamma cameras use a collimator to form a projected image of the incident photon flux by geometrically rejecting photons with oblique incidence, and only permitting acceptance of photons which conform to a narrow range of incident directions. By contrast, CA imaging systems generally accept a much larger fraction of the incident flux. When imaging a point source (with low statistical noise), in the far-field geometry, then each photon contributes to casting a shadow (encoded flux) of part of the aperture pattern onto the detector surface. This means that the counts of the point source spread over a non-point like surface area. The size of the aperture shadow depends on the distance of the point source from the CA. To obtain a useful image, the encoded pattern is then decoded, most often by using the correlation of the observed pattern with a suitable decoding function.

The basic mathematical formulations that describe the formation of the CA image when imaging a planar source is summarized below based on the analysis in [8]. For simplicity, consider the case of a 2D source emitting at distance $z$ from the detector, and encoded by a CA placed at distance $b$ from the detector. The 2D planar source object, at distance, $a=(z-$ $b$ ), from the CA, is denoted by array $\mathrm{O}$. The aperture pattern is given by the array A (a 2D function containing a zero where the aperture is opaque, and one where it contains a hole). With 
reference to Fig. 1 the distribution of recorded counts in the detector at position $\vec{r}_{i}$, is represented by the array $\mathrm{D}$ and is given by:

$D\left(\vec{r}_{i}\right)=\iint_{\vec{r}_{o}} O\left(\vec{r}_{o}\right) A\left(\frac{a}{z} \vec{r}_{i}+\frac{b}{z} \vec{r}_{o}\right) \Omega\left(\vec{r}_{o}, \vec{r}_{i}\right) d^{2} \vec{r}_{o}$

where:

$\Omega\left(\vec{r}_{o}, \vec{r}_{i}\right)=\frac{p_{m}{ }^{2}}{a^{2}} \cos ^{3}(\theta)$

where: $\theta=\arctan \left(\left|\vec{r}_{i}-\vec{r}_{o}\right| / z\right)$ and $p_{m}$ is the mask pixel size. The above theory has been shown to work well in low noise stellar applications [10] where the $\Omega\left(\vec{r}_{o}, \vec{r}_{i}\right)$ factor is constant (i.e. $\cos ^{3}(\theta) \cong 1$ ) and thus the imaging process can be considered to utilize a far-field geometry that provides artifact-free images.

In contrast, this is not the case in medical imaging applications where gaining close proximity to the object is crucial if acceptable sensitivity is to be achieved. In practice, this means that the distance, $b$, is typically $\sim 20-100 \mathrm{~cm}$, in which case the incident angle of gamma-rays photons is no longer approximately parallel and thus more oblique incidences are recorded than perpendicular incidences. This causes solid angle variation of the intensity of the projection of the mask on the detector. The consequence of the $\cos ^{3}(\theta)$ factor of expression (2) has been previously mathematically expanded into a Taylor series to second order [8]. Each of these expansion terms contributes various artifacts arising from the imaging geometry whose shape has been mathematically predicted, details appear elsewhere [8].

In the particular case of SM, the imaged region can be approximated to be a uniform volume of tissue with approximately uniform background activity, superimposed upon a small region of enhanced activity which is usually attributed to a lesion or disease presence. This target area is typically smaller than the sensitive imaging area provided on a clinical gamma camera. Displacement of the camera from the breast allows the projected flux to fill the camera Field of View (FoV) which, when reconstructed, can provide a corresponding projected resolution improvement due to image magnification. Solid angle losses in photon flux, which might otherwise occur if a collimator were used, are countered by the inherent wide angle acceptance of CA imaging, and the high open area $(\sim 50 \%)$ when MURA CAs are used. Furthermore, the displacement of the CA and the gamma camera away from close proximity to the breast means that shielding can be introduced to mitigate the effects of unwanted cardiac, liver, and bladder tracer uptake in other regions.

\section{GEOMETRY}

We have investigated several different masks, but here we only report on a $41 \times 41$ element MURA and $62 \times 62$ element NTHT placed at $30 \mathrm{~cm}$ away from the camera with the source object separated from the mask by $10 \mathrm{~cm}$. This imaging configuration ensures a magnification factor of four. The CA mask size is $8.2^{2} \mathrm{~cm}^{2}$ with each CA-hole defined as a square of size $0.2^{2} \mathrm{~cm}^{2}$. The imaging detector is defined by a $164^{2}$ pixel array corresponding to an imaging area of $38.2^{2} \mathrm{~cm}^{2}$.

Four different source objects were imaged. Initially we consider an on-axis point source, and then the size of the object systematically increases but with fixed thickness $(0.1$ $\mathrm{cm})$. These represent approximately planar (2D) square objects of different sizes ranging from $\left(1^{2}-12^{2} \mathrm{~cm}^{2}\right)$. Then the extension to a 3D source distribution is also considered with hot 3D source objects with a similar range of sizes as a planar objects but each having a fixed thickness $(6 \mathrm{~cm})$. Finally we emulate SM by imaging a hot $3 \mathrm{D}$ phantom of size $10 \times 10 \times 6$ $\mathrm{cm}^{3}$ with a spherical lesion of size 0.5 and $1 \mathrm{~cm}$ diameter, located within the phantom, with different Tumor-toBackground-Ratios (TBRs). The spherical lesion (tumor) was positioned at $3 \mathrm{~cm}$ depth from the surface of the breast.

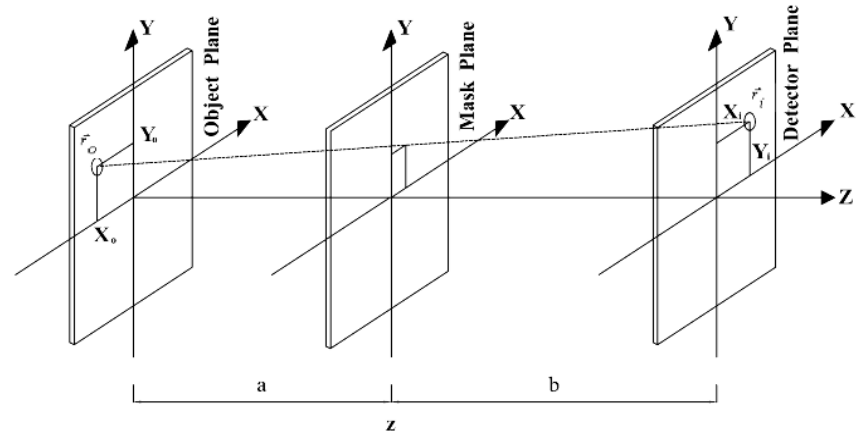

Fig. 1. A schematic representation of the CA geometry adapted from [8].

\section{METHODS}

A combination of three methods were undertaken to investigate the form of artifacts arising when imaging the aforementioned source object configurations. The first method is based on the well known MCS approach, the second method is based on Pseudo-Ray Tracing (PRT). This is based on purely calculating the angle of incidence of each point in the object that successfully strikes an open aperture element and then hits a detector element. The third method is based on a simple approach called Binary Mask Shift (BMS) representing the action of a distributed source in the projective CA imaging geometry. The latter method is attractive because this allows us to study these artifacts with, and without the effect of $\Omega\left(\vec{r}_{o}, \vec{r}_{i}\right)$ term of (1). The following subsections present and discuss these methods when imaging a variety of source objects. The section closes by describing near field correction techniques.

\section{A. Monte Carlo Simulation Method}

MCS using MCNPX code [11] (version 2.4.0) is used here to model the photon transport and detailed physical interaction of photons through the mask to the detector. The statistical uncertainty in position read out and in the recorded energy deposition process was also simulated post-simulation by inhouse Matlab code. Thus, all the major physics aspects of the imaging system are considered. Further details of the MCS are available in [4] and references therein. 
All simulations were initially undertaken using 2D source CA detector geometry with a $140 \mathrm{keV}$ gamma-ray emitter source using the above camera configuration geometry. The CA mask is a tungsten sheet of thickness $0.15 \mathrm{~cm}$. This provides an overall $\sim 50 \%$ transmission of the entire incident flux, as $99.4 \%$ of the photons incident on the tungsten component will be attenuated. In addition, a $0.15 \mathrm{~cm}$ tungsten frame surrounds the CA was considered to minimize detection of oblique photons arriving from outside the FoV.
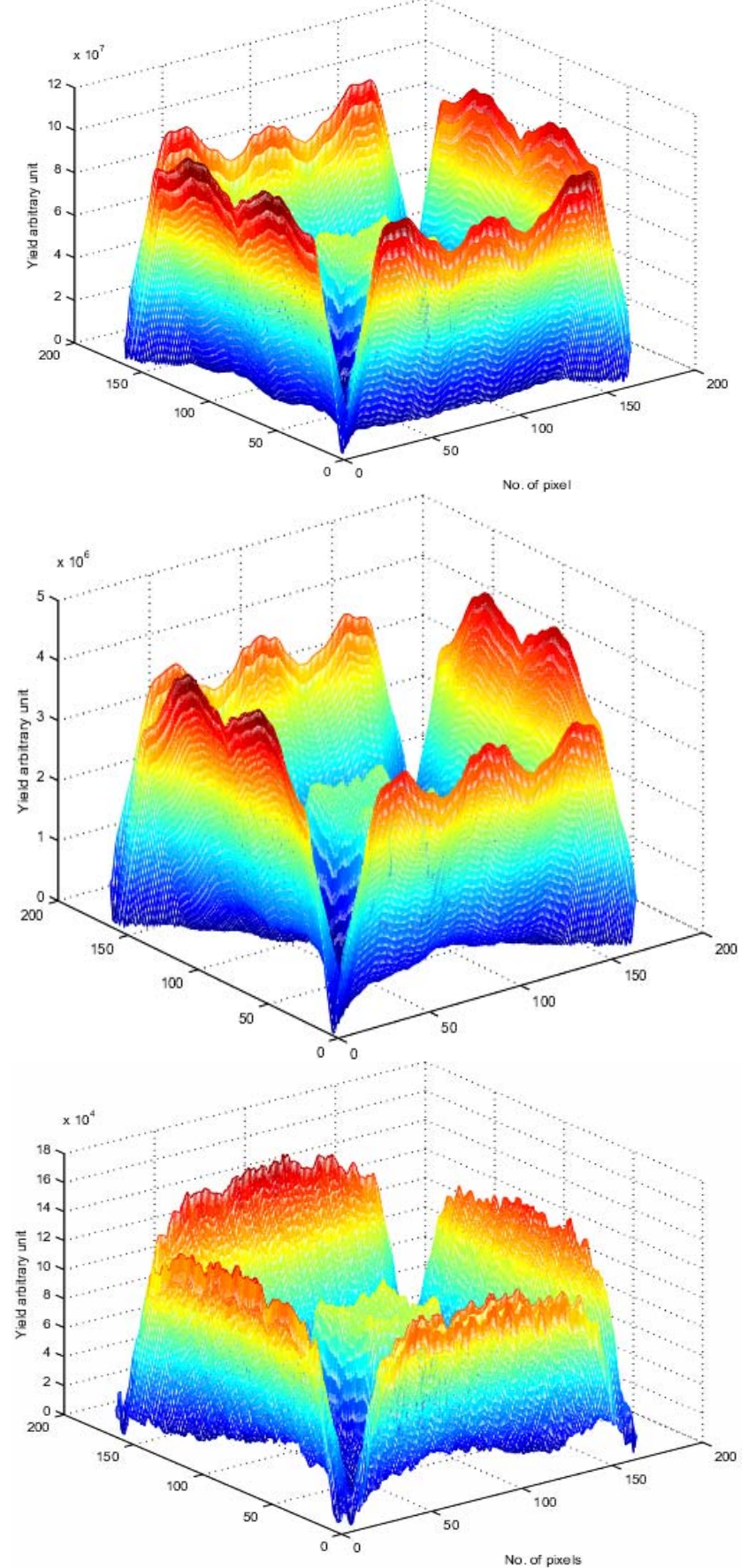

Fig. 2. Exemplar of the predicted flat field distortion from the BMS method (top) compared to the PRT method (middle) and MCS method (bottom) of a planar square source of $3^{2} \mathrm{~cm}^{2}$. This demonstrates the effect of the solid angle factor as it causes a sensitivity dip toward the center.

\section{B. Binary Mask Shift Method}

This method was initiated using the projected image of a perfect MURA (or pattern based on MURA) binary mask generated by an on-axis point source. Then using assumed knowledge of the shape of the distributed source to be imaged, the projected pattern was systematically shifted according to the projected point source pattern that would be obtained by every non-zero element in array $O$. The result of each single or "binary shift" of the projected pattern is then summed with the preceding projection pattern. These summed shifted copies were decoded with the usual post-processing, $\mathrm{G}$ function (an inverse filter of $A$ ). This binary mask displacement method represents a far-field approximation and is also given by using (1) but with a constant $\Omega\left(\vec{r}_{o}, \vec{r}_{i}\right)$ term. This noise-less composite method was used to demonstrate the effect of artifacts from planar objects or displaced (shifted) sources.

\section{Pseudo-Ray Tracing Method}

This method has been used to investigate the background distortion observed when imaging planar and 3D source phantoms. The projection of a CA-hole cast by a point on the object falling on the detector grid is represented by (1) and is calculated using purely geometric formulae. Simple in-house code was written to do this calculation based on calculating the solid angle [12] subtended by each element of the mask whose shadow projected along the flux direction is intercepted by the detector. This obviously requires a-priori knowledge of the source distribution. In SM this is not a major issue as the breast is commonly compressed to a known thickness, and the 2D projection can be obtained from a simple optical camera. The code finds the center of the projection of the mask-hole by ray-tracing.

Mask transparency, finite mask thickness and statistical noise are not considered in these two initial approaches. But the projection data were convolved with a $2 \mathrm{D}$ Gaussian of standard deviation, $\sigma=1.57 \mathrm{~mm}$, to simulate the intrinsic PSF blurring of the gamma camera.

To explore the effect of $\Omega\left(\vec{r}_{o}, \vec{r}_{i}\right)$ term the reconstructed image of a planar square source of size $3^{2} \mathrm{~cm}^{2}$ from the PRT method were compared to the corresponding from BMS and MCS data and exemplar surface plots are shown in Fig. 2. Both methods successfully predicted the flat field background distortion artifacts of the uniform 2D object imaged, similar to that using MCS.

\section{Near-field Artifacts Corrections}

In the following we present results from three near field corrections. These methods are solid angle correction, mask/anti-mask correction and background subtraction. The solid angle correction is equivalent to a zero order correction where as the mask/anti-mask technique is equivalent to a second order correction as referred to in ref. [8]. These correction techniques were applied to a full 3D SM data using CAs (see section V). 


\section{Solid angle correction}

Near field geometry causes solid angle variation (modulation) of the intensity of the projection of the mask on the detector dependent on the position of the source as it projects photons through the aperture. The projected image can be corrected and this near field effect can be compensated from the knowledge of the distance $z$. This can be achieved if the projected image, $D$, is divided by $\Omega\left(\vec{r}_{o}=0, \vec{r}_{i}\right)$ see Fig. 3 .

This only corrects the incident solid angle of gamma-rays photons seen by a source at the center of the mask i.e. as if the reconstruction was focused at the center of the FoV. In other words, if we were to image an idealized point source centered on-axis of the FoV and then applied this correction to the projected image before decoding, it will flatten the field. If the corrected image were then decoded with the post-processing array, $\mathrm{G}$, it would produce a perfect response function image with zero side-lobes. This is the ideal response from a point source and thus represents the far-field case.
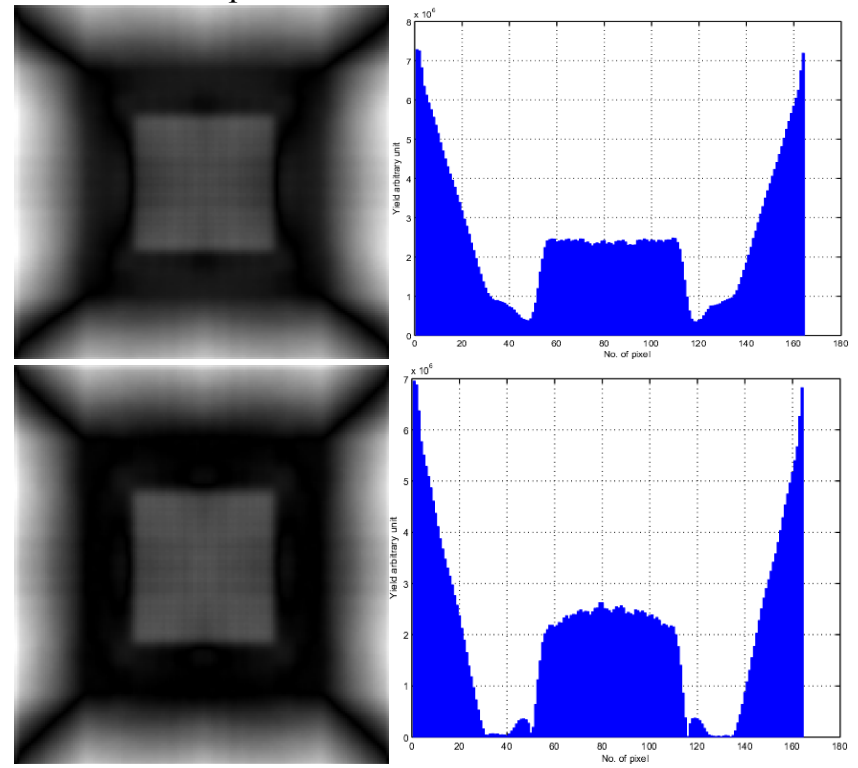

Fig. 3. The reconstructed image of the predicted flat field distortion of planar square source ( of $4^{2} \mathrm{~cm}^{2}$ ) before solid angle correction (top left) with a vertical profile through center plot (top right) and after solid angle correction (bottom left) with similar vertical profile (bottom right).

\section{The Subtracted Flat Field Correction}

As will be demonstrated in the results section, the raw projection images of the 3D CA-SM data need to be corrected from the effect of volumetric background corruption artifacts. One way to achieve this is through using the PRT method. In this case we generate a prediction of the unwanted background structure by simulating a uniform 3D object of similar size to the breast phantom, and then do a least squares fit to the observed data. A simple background subtraction technique is performed to remove the resulting scaled background. This technique is important as it can subtract out background contributions from activity above/below and within the decoded plane under consideration containing the target object (lesion).

\section{RESULTS AND DisCUSSION}

\section{A. Imaging a 2D Source Object}

Imaging an on-axis point source using the discussed geometry, projects a shadowgram of the mask that covers the entire detector area. This however, produces unwanted small cross-shaped side-lobe artifacts in the decoded image. Here we consider the effects of imaging planar square source objects with variable size. Each point source of the object produces its own projected shadow of the CA. The coded image (the recorded $2 \mathrm{D}$ distribution shadowgram) is the sum of each of these point source projections. Our investigation, using MCS, demonstrated that once the object increases in size $9 \mathrm{~cm}^{2}$ the non-uniform background distortion also re-enforced and we tend to gradually loose the objects as the artefact dominates the image. In other words, different object sizes produce different shapes of artifact in the reconstructed image.

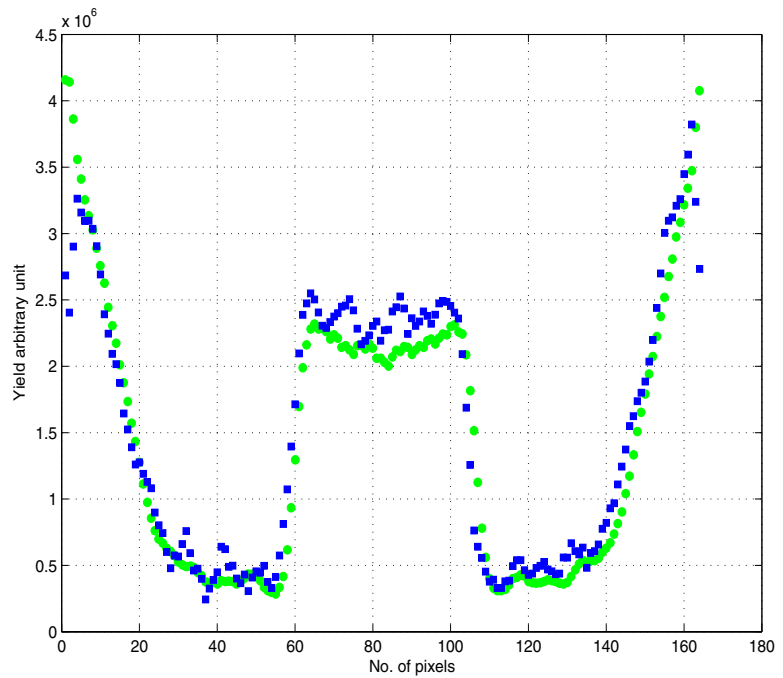

Fig. 4. Exemplar vertical profiles of predicted flat field distortion for 2D source object of $3 \times 3 \mathrm{~cm}^{2}$ from PRT method (filled circle) compared with the corresponding produced by 2D MCS (filled square). The slight discrepancies remaining are due to photon statistics and MCS geometry specific effects such as mask thickness.

In an attempt to investigate the background distortions appearing in the reconstructed image, the aforementioned BMS method was used. This method produces a background distortion artifact that is due to the finite size of the object (off-axis sources) but ignoring other artifacts due to $\Omega\left(\vec{r}_{o}, \vec{r}_{i}\right)$. To consider the effect of all geometrical artifacts a PRT method is used and found to predict flat field distortions and produces a similar result to the MCS data as shown in Fig. 4. The source of this non-uniform background noise may be due to a combination of several factors: 1) the off-axis sources as the object is no longer-like a point source as it has an extended size, 2) the inherent non-linearity in the photon flux impinging on the detector, and 3) incomplete (partial coding) of the mask pattern due to finite size of the detector.

\section{B. Imaging a 3D Source Object}

Extending from 2D planar to 3D volumetric source objects means that equation (1) is now extended to triple integrals that 
further increase the complexity of the decoding procedure. This is because we can, in practice, only focus in one plane and the other underlying/overlying planes potentially contribute to blurred out-of-plane artifacts. We therefore investigate the effects of imaging artifacts arising from 3D uniform objects of fixed thickness $(6 \mathrm{~cm})$ but have different target volumes ranging from $6-864 \mathrm{~cm}^{3}$.

We also consider the difference between a full 3D PRT prediction of the background compare to just considering 2D in-plane effects. The flat field distortion artifacts were also visualized and appear some how similar to that observed when imaging a 2D planar object. Fig. 5 demonstrates this by showing exemplar profiles of the decoded image of the predicted flat field distortion obtained from the PRT method for 2D planar and 3D volumetric source objects. This suggests that the source of gross artifacts is largely attributed to the effect of in-plane distributed radioactivity and the effect of incomplete (partial coding) of the mask pattern due to off-axis sources.

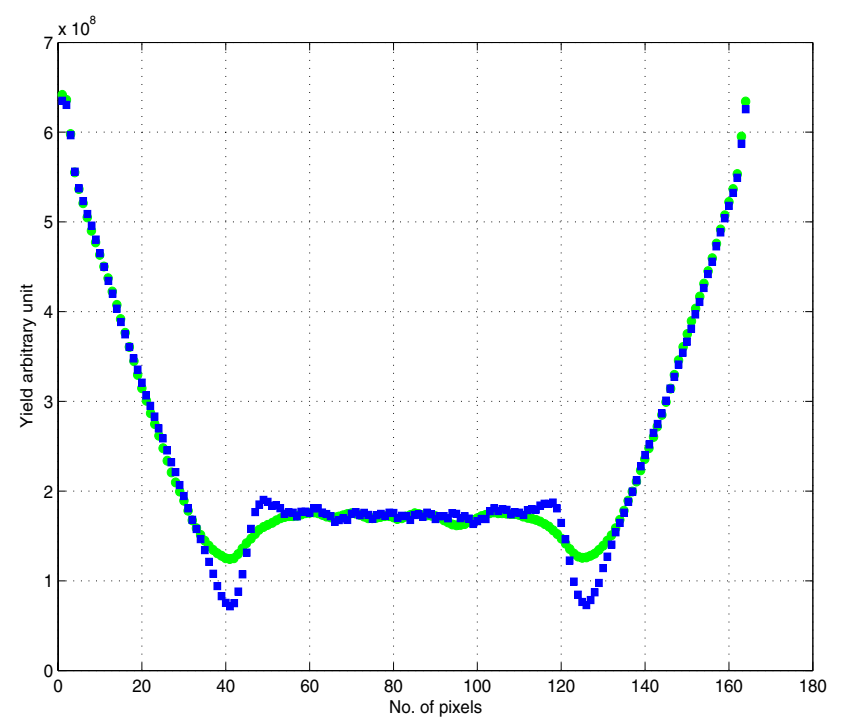

Fig.5. Exemplar vertical profiles of predicted 2D flat field distortion from the PRT method for a planar source of $5 \times 5 \mathrm{~cm}^{2}$ (filled square) compared with 3D source object of $5 \times 5 \times 6 \mathrm{~cm}^{3}$ (filled circle) after least squares fit.

The 3D set of investigations suggests that the main cause of the distortion artifacts, in near field geometry, is the finite distributed size of the source object mainly from within the focal plane. It also suggests that the form of artifacts, in term of the shape and the magnitude of the side-lobes, arising from such imaging geometry can be predicted. These are very encouraging results and suggest that the MURAs-CA near field distortion observed with distributed 3D source object, as might be found in SM, can be easily predicted and corrected. Some preliminary investigations have been carried out in the next section.

For faithful object decoding there is a need for complete encoding of a full mask cycle on the detector. This can be achieved by using a much larger detector with a mosaic mask $(2 \times 2$ of the basic mask pattern) [7]. This is an attractive option and has been investigated using the PRT method with a planar 3D source object of variable sizes. Our investigations (omitted here) using a mosaic of the basic pattern of the masks used here and a planar source (of variable sizes) suggests that this technique preserves the object and provides a uniform sensitivity a cross the fully coded FoV. In practice, the standard clinical gamma camera detector is limited. Taken together with the nee to shield the detector from non-specific (non-breast) activity means that the use of a mosaic mask may be impractical.

\section{Imaging a Bright Sphere in a warm Background}

The main task in SM imaging is to be able to detect a lesion in a general non-zero noisy background. The presence of a hot background phantom offsets, in part, the magnification advantages of CA-SM and thus seriously confuses the diagnostic processes. Fortunately, from the above discussion the form and structure of the artifacts have been successfully predicted and thus, can now be eliminated or minimized. The imaging performance of the CA-SM system in resolving a lesion embedded in a hot $3 \mathrm{D}$ phantom of $10 \times 10 \times 6 \mathrm{~cm}^{3}$ produced from PRT method was investigated. This investigation demonstrates the intrinsic capability of the CA imaging in detecting a signal in a hot background. In particular, we demonstrate how post-acquisition near field image corrections can improve object detection in terms of CNR and resolution. Note, the "noise" present arises due to systematic effects within the correlation process. We consider these calculations for both different lesion sizes $(0.5 \& 1 \mathrm{~cm}$ diameter) and a variety of imaging TBRs conditions ranging from 5:1-100:1.

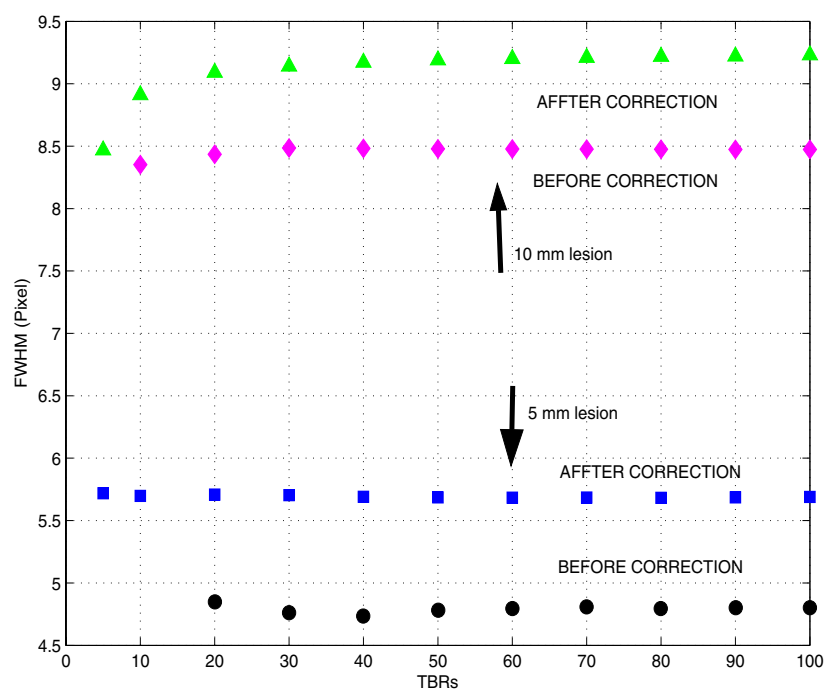

Fig.6. shows tumour FWHM as a function of TBRs before and after near field corrections. Values calculated from 3D data images of 5 and $10 \mathrm{~mm}$ lesions. Note that in each case, projection and partial volume effects reduce the measurement to less than the actual lesion size, and that it appears that TBRs has relatively little effect on observed resolution above TBR=20. NB: 1 pixel $=2 \mathrm{~mm}$, and due to imaging geometry a magnification of 3 is also present

The observed object size expressed in terms of Full Width Half Maximum (FWHM) of 5 and $10 \mathrm{~mm}$ diameter lesions was quantified before and after the near-field corrections, with the results given in Fig. 6. Values were averaged based on 4 profiles (horizontal, vertical and the two diagonal directions) 
drawn through the center of the lesion after a Gaussian curve fit to the data points. The CNR in this work is defined as the difference between the signal, $h_{2}$, and the mean background values, $h_{l}$, divide by the average variation in the background, $\sigma_{1}$, (as the number of pixels in the object were too few to be statistically significant or unbiased) as:

$$
C N R=\frac{h_{2}-h_{1}}{\sigma_{1}}
$$

The mean of the background is obtained using a region of interest (ROI) defined by a square area that is six times the FWHM. Fig. 7 illustrates these data, demonstrating that after the near-field corrections we are able to visualize a $10 \mathrm{~mm}$ lesion down to a TBR of 5:1 and a $5 \mathrm{~mm}$ lesion down to TBR of 10:1. Prior to near field corrections the limits of visibility were at TBRs of 10:1 and 20:1 respectively. From these data we conclude that the use of near-field corrections in CA-SM system has relatively small effect on lesion resolution, but however, it does significantly improve the system contrast by approximately a factor of 10 .

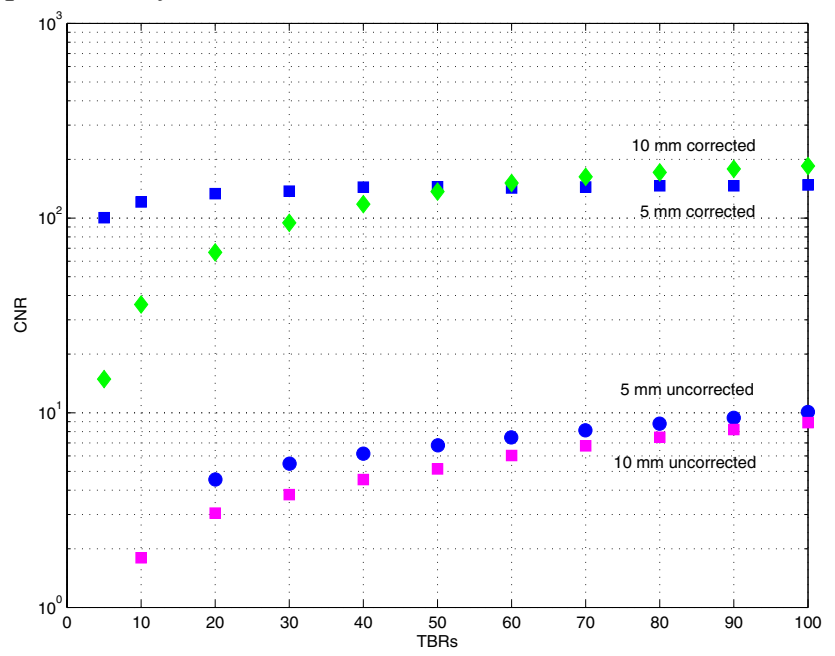

Fig.7. Tumor CNR as a function of TBRs before and after near-field corrections for 10 and $5 \mathrm{~mm}$ lesion. Values calculated from Eq. 3 for images produced from PRT method.

\section{CONCLUSION}

Image artifacts are intrinsic to $\mathrm{CA}$ imaging when using near-field geometry even when imaging small objects such as a point source. Gross distortions artifacts were visualized for objects larger than $1 \times 1 \mathrm{~cm}^{2}$. The magnitude of these artifacts further increase once the object size increases, resulting in loss of information for the off-axis sources. Along with the effect of the in-plane distributed radioactivity, this affects the decoding process, thus attributed to the appearance of undesirable artifacts. Similar artifacts were visualized when imaging much thicker 3D objects. The combination of presented here methods provide valuable insight in understanding and predicting these imaging artifacts. We have also demonstrated that applying near-field correction techniques using PRT to the CA-SM data has minimized the magnitude of these artifacts, and helps preserve the target object size and imaging contrast.

\section{ACKNOWLEDGMENT}

M. A. Alnafea gratefully acknowledges financial support from King Saud University, Saudi Arabia.

\section{REFERENCES}

[1] R. F. Brem , AD Kieper, J. A. Rapelysea, and S. Majewski,. "Evaluation of high-resolution, breast specific, small-field-of-view gamma camera for the detection of breast cancer", Nucl. Instrum. and Meth A 497 pp. 39-45, 2003.

[2] B. Mueller, M.K. O'Connor, I. Blevis, D.J. Rhodes Smith R, Collins DA et al, "Evaluation of a small cadmium zinc telluride detector for scintimammography", J. Nucl. Med. vol. 44, no. 4, pp 602-609, 2003.

[3] R. Pani, G. De Vincentis, F. Scopinaro, et al., Dedicated gamma camera for single photon emission mammography (SPEM)," IEEE Trans. Nucl. Sci., vol. 45, pp. 3127-33., 1998.

[4] M. A. Alnafea, K. Wells, N.M. Spyrou, M.I Saripan, M. Guy, and P. Hinton., "Preliminary results from a Monte Carlo study of breast tumour imaging with low energy high-resolution collimator and a modified uniformly-redundant array-coded aperture", Nucl. Instrum. and Meth A 563 pp. 146-149, 2006.

[5] M. A. Alnafea, K. Wells, N.M. Spyrou, and M. Guy, "Preliminary Monte Carlo study of coded aperture imaging with a CZT gamma camera system for scintimammography", Nucl. Instrum. and Meth A accepted for publication.

[6] E. E. Fenimore. "Coded aperture imaging: predicted performance of uniformly redundant arrays", Appl. Opt., vol. 17, no. 22, pp.3562-3570, 1978.

[7] E.E. Fenimore and T. M. Cannon, "Coded aperture imaging with uniformly redundant arrays", Appl. Opt., vol. 17, no. 3, pp. 337-347, 1978.

[8] R. Accorsi, F. Gasparini, and R. C. Lanza, "Near-field artifact reduction in coded aperture imaging", Appl. Opt., vol. 40, no. 26, pp 4697-4705, 2001.

[9] O.I. Vassilieva and R. C. Chaney, "Method for reducing background artifacts from images in single-photon emission computed tomography with a uniformly redundant array coded aperture" Appl. Opt., vol. 41, no. 7, pp. 1454-1461, 2002.

[10] Caroli, E., Stephen, J. B., Di Cocco, G., Natalucci, L., and Spizzichino, A., "Coded aperture imaging in x- and gamma-ray astronomy", Space Science Reviews, 45, 349-403, 1987.

[11] J.F. Briesmeister, Ed., "MCNP - A General Monte Carlo N-Particle Transport Code, Version 4B," LA-12625-M, 1997.

[12] H. Gotoh, and H. Yangi (1971). "Solid angle subtended by a rectangular slit". Nucl. Instrum. and Meth in physics reasach 221:56-59. 